\title{
X-ray image intensification in ruminant physiology
}

\author{
By G. Wenham, The Rowett Research Institute, Bucksburn, Aberdeen AB2 $9 S B$
}

The general introduction of the X-ray image intensifier (II) over the last decade has opened up new possibilities for X-ray examination. The brightness of the II, which is several thousand times higher than the conventional fluorescent screen, allows the image to be viewed in normal room lighting. If a television chain is connected, the operator has control over contrast and brightness to further enhance visualization of detail and the image can be stored electronically on magnetic tape or disc, or on an image storage tube. This increased brightness and detail allows photographic exposures of the output screen to be obtained with a radiation dose to the subject 5 -10 times lower than that required for conventional radiography using intensifying screens. This, together with approximately a further one-third reduction in the continuous viewing dosage, is an important safety consideration (Gudden, Hofmann \& Marhoff, I972).

\section{Equipment}

The II is an evacuated glass tube, at one end of which is a fluorescent screen, usually consisting of zinc-cadmium sulphide. X-rays spatially modulated by the subject impinge upon the fluorescent screen, thereby converting the $\mathrm{X}$-ray pattern into a visible image. In optical contact with the fluorescent screen is the photo-cathode which emits electrons on its free side in direct proportion to the varying light intensity from the fluorescent screen. These photo-electrons are accelerated across the II by an increasing positive potential, approximately $25 \mathrm{kV}$, and electronically focused to bombard the viewing screen or output phosphor at the other end of the tube (See Fig. I). Because the photo-electrons have been accelerated by the high potential difference, their kinetic energy has been increased, so that any single electron may generate thousands of light quanta in the viewing screen. Because the viewing screen is smaller than the photo-cathode, the electron stream is concentrated and this also improves the brightness by increasing the light flux per unit area. This reduced image may be viewed directly through an optical system, photographed on cine-film or cut film, or, as is more usual, scanned by a television camera and displayed on a monitor. The facility of videotape recording is of inestimable value; each examination, once recorded, can be viewed immediately to assess the results. The sequence can be examined time after time to obtain more information, and each aspect of the sequence, movement and position, can be concentrated upon in turn. The tape can be wiped clean and re-used, stored as a record, or the image may be transferred to $16 \mathrm{~mm}$ cine-film. Until quite recently the television chain augmented 


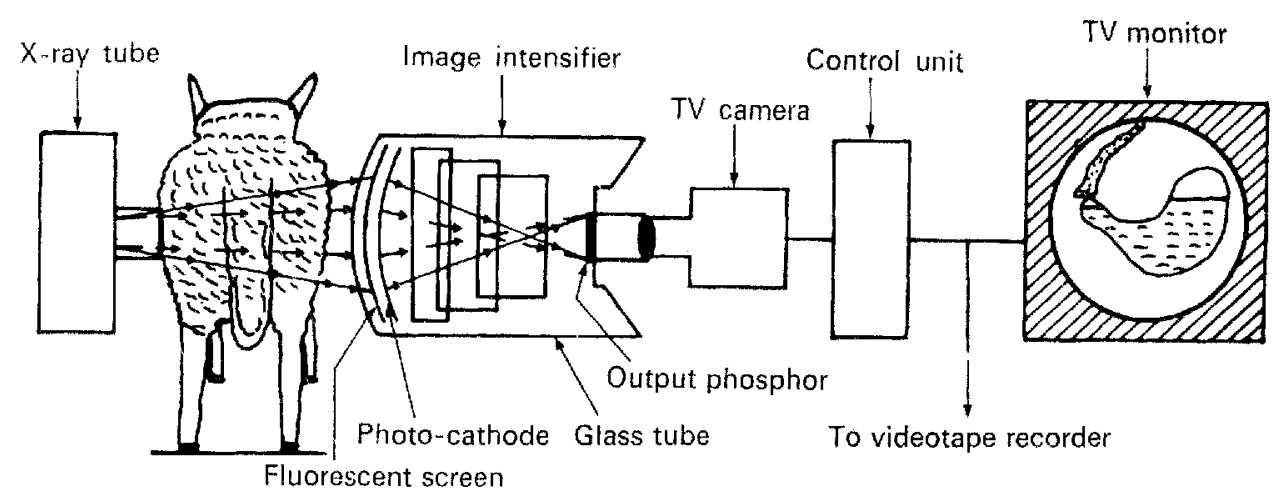

Fig. I. X-ray image intensifier with television monitoring.

by radiography produced the best results regarding fine detail, but with the new generation of high-resolution image intensifiers, fluorography, i.e. photography of the II viewing screen, is considered to give the best results (Pfeiler \& Linke, I972).

'The equipment used at this Institute is: a Biangulix rapid X-ray tube with I $\mathrm{mm}$ and $0.6 \mathrm{~mm}$ focal spots, and a Pantoskop 3 tilting fluoroscopic table with a fully automatic explorator, on which is mounted a Sirecon 2 ro in $\times 6$ in image intensifier with closed-circuit television fitted with automatic brightness stabilization, made by Siemens of Germany. The X-ray generator is a $200 \mathrm{kV}, 1000 \mathrm{~mA}$ Triplex Optimatic made by Elema-Schönander of Sweden. The videotape recorder is a Sirecord $\mathrm{X}$ I in helical scan made by Siemens.

\section{Examinations}

Many different kinds of investigations lend themselves to the use of an II. It is especially valuable when it is wished to define a rapid and complex sequence of movement, such as the contractions of the digestive tract and the consequent movement of digesta, for radiographs of such events provide at best a disjointed sequence. Muscular activity and some of its mechanical consequences can be recorded by use of electrodes, pressure recorders and strain gauges, but these do not indicate one of the most important functions of gut motility, namely the propulsion of digesta.

Some of these examinations which have been carried out on ruminants and pigs at the Rowett Research Institute are: (i) examination of the mouth, oral pharynx and oesophagus and oesophageal groove in sucking lambs and kids; (2) contractions of the reticulo-rumen; (3) movements of gas from the rumen and abomasum; (4) contractions of the abomasum, with particular reference to the pyloric antrum and the pyloric constriction, in relation to the activity of the duodenal bulb; (5) the passage of digesta through the duodenum, jejunum and ileum, and activity of the caecum;

Lateral radiograph of the barium-filled abomasum and duodenal bulb of a sheep, showing pyloric antrum contractions. The outline of the omasum can just be seen. The animal had received $500 \mathrm{ml}$ of barium sulphate--milk from a bottle. 
Proceedings of The Nutrition Society, Vol. 33, No. 2.

Plate I

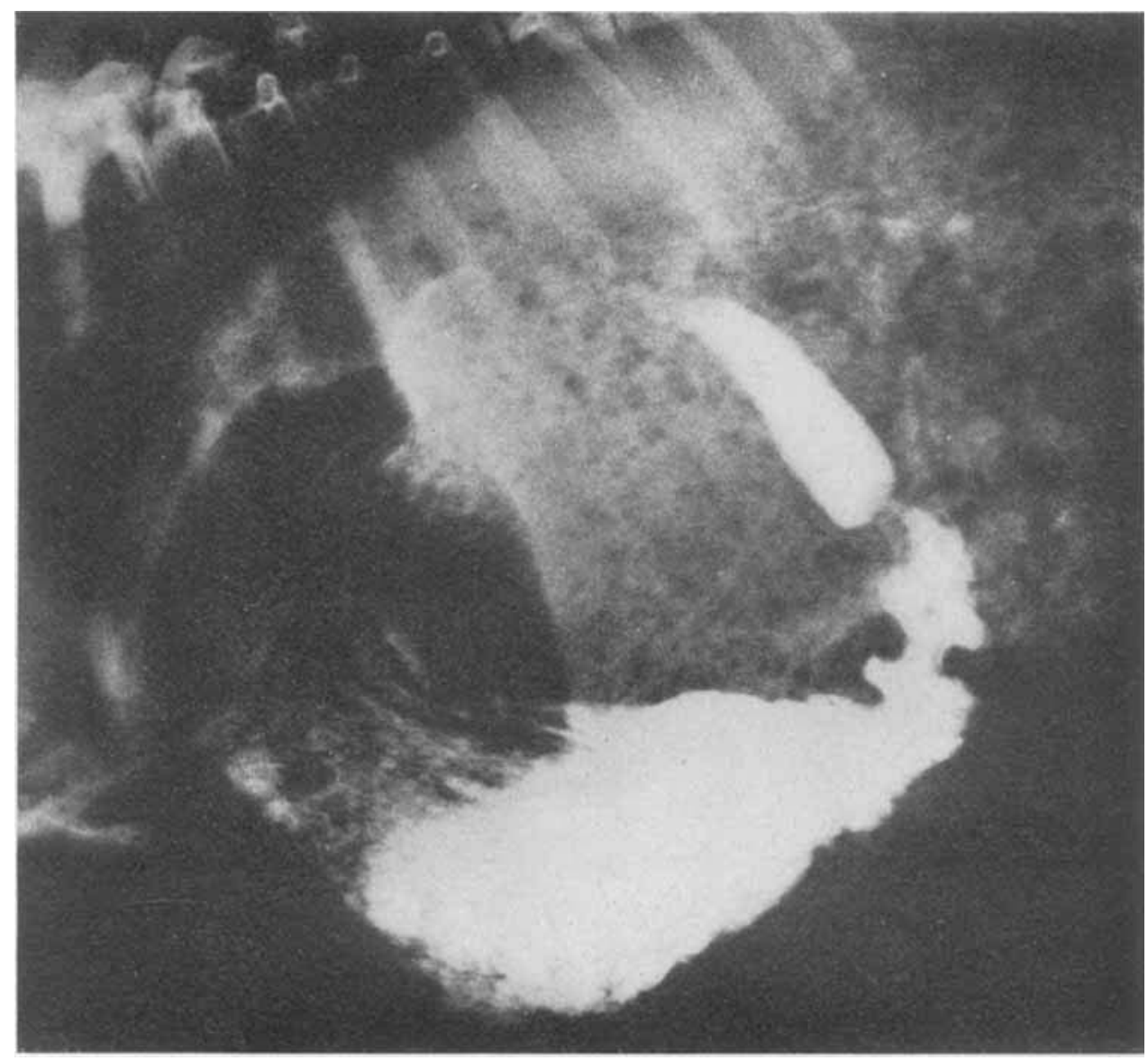

\section{G. WENHAM}


(6) orthopaedic examinations for trauma or pathology; (7) kidney function and bladder catheterization; (8) positioning of intra-vascular catheters during abdominal surgery and selective intra-vascular catheterization either percutaneous or by simple section; (9) lymphography to determine the course of lymphatic vessels; (10) biopsy sampling of abdominal viscera.

Some examples of these examinations were demonstrated to the Society on videotape, but some selected applications are enlarged upon here.

\section{Abomasum, pylorus and duodenal bulb}

A I :I (v/v) mixture $(500 \mathrm{ml})$ of milk and barium sulphate in suspension (Micropaque; Damancy Co.) was sucked from a bottle by a lamb or kid goat. This effectively

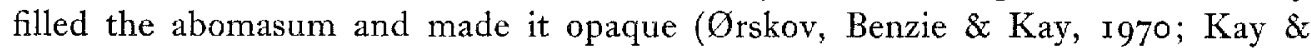
Ruckebusch, 1971; Kay, Ørskov \& Wenham, 1972). The barium sulphate usually settled to the greater curvature and reached the pylorus very soon after administration, while the other contents were displaced into the cardiac region. The abomasum then contained three components, gas, unmarked fluid and barium-marked fluid, with clear level interfaces between them. The fundus of the abomasum acted as a largely inactive reservoir of digesta and the contractions commenced a little over half-way to the pylorus. In the pyloric antrum the contractions were very strong, but contractions of the circular muscle only had a propulsive effect within the last IOO-I $50 \mathrm{~mm}$. As the contractions did not completely close the lumen of the antrum, thereby isolating the region distal to them, some digesta flowed retrogradely and some into the duodenum. The amount flowing to the duodenum depended both on the propulsive activity of the pyloric antrum and the extent to which this was opposed by constriction of the pyloric sphincter. The pyloric constriction was often open when the antral contractions were some distance away, allowing digesta to enter the duodenal bulb. The pylorus usually closed as the contractions approached more closely, and the contractions ended there with no concomitant contractions of the duodenal bulb. The bulb appeared to have a pattern of activity which was distinct from that of the pyloric antrum and also from that of the distal duodenum. The bulb may contract after the bolus from one antral contraction partly fills it, or it may contract only after several boluses have contributed to its filling. When filled, the bulb appears remarkably distended, having two or three times the diameter of the more distal parts of the duodenum. (Sce Plate 1 ). Some contractions of the bulb, often originating a short way beyond the pylorus, propel part of the contents of the bulb $100-150 \mathrm{~mm}$ into the duodenum, but the wave of contraction is not sustained and the contents fall back into the bulb. When a true peristaltic contraction originates in the duodenal bulb, usually close to the pylorus, the whole of the contents are propelled fairly slowly along the ascending duodenum and into the hepatic sigmoid flexure, but once the flexure is passed the speed of passage increases very rapidly, certainly in young animals, the bolus passing into the jejunum at a rate of 200-300 $\mathrm{mm} / \mathrm{s}$ and continuing through the jcjunum at approximately half this speed (Kay et al. 1972). 


\section{Orthopaedic examination}

The use of an II for skeletal examinations is one which is not often employed, but it can save a lot of time, effort and money. It has been used on horses and cattle (Knezevic, 1972) and an II is in use at the Equine Research Centre, Newmarket. If a sheep, or other animal, presents clinical signs of skeletal injury or pathology and the cause is not immediately apparent, it can be a costly and time-consuming exercise to carry out a general skeletal survey radiographically, often using a number of large X-ray films, and involving a lot of animal manipulation. An easier approach is to scan systematically the whole skeleton under the II and take a radiograph only of doubtful areas. A case in point will illustrate this. A Finnish Landrace ram was referred for examination with paralysis of both hind limbs, and showing no tendon or pain reflexes. The animal was scanned under the 11 , commencing at the hips. No abnormality was found in the femora or pelvis, some arthritic changes in the lumbar vertebrae, but the thoracic vertebrae showed ossifying and ankylosing spondylosis with an oblique fracture of vertebra $T_{I I}$. The whole examination and satisfactory diagnosis was greatly facilitated by the II.

\section{Intra-vascular catheters}

With the current interest in the measurement of blood flow, and in blood sampling from, or infusion into, specific arteries and veins, the requirement for the introduction of intra-vascular catheters is increasing (White, Wenham, Hughes, Mathieson \& Chalmers, 1969; Mason \& White, 1971; Webster \& White, 1972, 1973). The validity of any results from such a preparation requires accuracy regarding the placing of a catheter or thermocouple. By slowly infusing a water-soluble contrast medium (e.g. Urografin $76 \%$; Schering) through the catheter the whole length of the catheter may be seen, or alternatively a radio-opaque catheter may be used and, by a forced infusion, the surrounding blood vessels can be seen in relation to the catheter. For example, if a catheter is inserted into one of the veins contributing to the hepatic portal vein of a sheep, the catheter directed downstream, a forced infusion would show first a single column of contrast medium where the vein is single or has only minor contributaries, then as it nears the porta the flow of contrast medium becomes broken and turbulent where the large rumen and gastric veins are confluent. Such observations permit one to assess whether a cool saline solution infused through a catheter in a mesenteric vein has mixed sufficiently with the blood flowing past a thermocouple placed in the porta to allow a thermodilution technique to be used for the assessment of hepatic portal blood flow (Webster \& White, 1972, 1973). Contrast medium infused into an aortic catheter can be seen to pulsate as it flows from the catheter tip, flowing caudally during left ventricle systole, and slightly cephalad during diastole.

Preformed radio-opaque catheters can be manipulated under the II to selectively catheterize blood vessels quite remote from the point of insertion. The catheter can be advanced, withdrawn or rotated, and by advancing or withdrawing an internal flexible guide-wire the catheter can be straightened or allowed to bend, to select 
the vessel required. A forced infusion of contrast medium will then outline the vessel entered. It is possible to catheterize a renal artery via a carotid or femoral artery, or to catheterize an hepatic vein via the jugular, thus eliminating the need for open abdominal surgery. With modern equipment such as television subtraction, image storage tubes, tapes and colour subtraction, the results from angiography are greatly improved (Gebaur, Lissner \& Schott, 1972).

\section{REFERENCES}

Gebaur, A., Lissner, J. \& Schott, O. (1972). Med. Prog. Technol. I, I 5.

Gudden, F., Hofmann, F. W. \& Marhoff, P. (1972), Med. Prog. Technol. 1, 104.

Kay, R. N. B. \& Ruckebusch, Y. (1971), Br. F. Nutr, 26, 30r.

Kay, R. N. B., Orskov, E. R. \& Wenham, G. (I972). F. Physiol, Lond. 227, 2P.

Knezevic, P. F. (1972). Acta radiol. Suppl. 319, 55.

Mason, V. C. \& White, F. (1971). F. agric. Sci., Camb. 77, 91.

Ørskov, E. R., Benzie, D. \& Kay, R. N. B. (1970). Br. 7. Nutr. 24, 785 .

Pfeiler, M. \& Linke, G. (1972). Electromedica 4, 2.

Webster, A. J. F. \& White, F. (1972). F. Physiol., Lond. 227, ${ }_{4}$ P.

Webster, A. J. F. \& White, F. (1973). Br. F. Nutr. 29, 279.

White, F., Wenham, G., Hughes, A. D., Mathieson, J. \& Chalmers, M. I. (1969). Proc. Nutr. Soc. 28, $60 \mathrm{~A}$. 